\title{
STONELEY WAVES IN A NON-HOMOGENEOUS \\ ORTHOTROPIC GRANULAR MEDIUM UNDER \\ THE INFLUENCE OF GRAVITY
}

\author{
S. M. AHMED
}

Received 21 May 2005

The aim of this paper is to investigate the Stoneley waves in a non-homogeneous orthotropic granular medium under the influence of a gravity field. The frequency equation obtained, in the form of a sixth-order determinantal expression, is in agreement with the corresponding result when both media are elastic. The frequency equation when the gravity field is neglected has been deduced as a particular case.

\section{Introduction}

Problem of Stoneley waves play an important role in the earthquake science, optics, geophysics, and plasma physics. Many authors such as Abd-Alla and Ahmed [1, 2], El-Naggar et al. [8], Das et al. [6], and others studied the effect of gravity of the propagation of surface waves (Stoneley waves, Rayleigh waves, and Love waves) in an elastic solid medium. Goda [9] studied the effect of inhomogeneity and anisotropy on Stoneley waves.

The study of granular medium has been necessiated by its possible application in soil mechanics, geophysical prospecting, mining engineering, and so forth. The theoretical outline of the development of the subject from the mid-1930s was given by Paria [13]. The present paper, however, is based on the dynamics of granular media as propounded by Oshima $[11,12]$.

The medium under consideration is discontinuous such as one composed numerous large or small grains. Unlike a continuous body, each element or grain cannot only translate but also rotate about its centre of gravity. This motion is the characteristic of the medium and has an important effect upon the equation of motion to produce internal friction. It is assumed that the medium contains so many grains that they will never be separated from each other during the deformation and that the grain has perfect elasticity. The propagation of Rayleigh waves in granular medium was given by many authors such as Bhattacharyya [5], El-Naggar [7], Ahmed [4], and others. In [3], Ahmed discussed the influence of gravity on the propagation of Rayleigh waves in granular medium.

This paper is devoted to the study of the effect of granular body and also of the gravity field in the propagation of Stoneley waves. The wave velocity equation has been derived in the form of a sixth-order determinant. The roots of this equation are in general complex 
and the imaginary part of an appropriate root measures the attenuation of the waves. It is shown that the frequency of Stoneley waves contains terms involving the acceleration due to gravity and so the phase velocity changes with respect to this acceleration due to gravity. When the gravity field is neglected, the frequency equation has been deduced as a particular case. Also when both media are elastic, the frequency equation reduces to the corresponding result obtained by Abd-Alla and Ahmed [2] in the form of a fourth-order determinant.

\section{Formulation of the problem}

Let $M_{1}$ and $M_{2}$ be two non-homogeneous orthotropic granular media. They are perfectly welded in contact and are under the influence of gravity. These two media extend to infinitely great distance from the origin and are separated by a plane horizontal boundary and $M_{2}$ is to be taken above $M_{1}$. Let $O x_{1} x_{2} x_{3}$ be a set of orthogonal Cartesian coordinates, the origin $O$ being any point on the plane boundary, $x_{3}$-axis is vertically downwards into the medium $M_{1}$.

We consider the possibility of a type of wave traveling in the direction $O x_{1}$, in such a manner that the disturbance is largely confined to the neighborhood of the boundary which implies that the wave is a surface wave.

Notice that at any instant all particles in any line are parallel to $O x_{2}$, having equal displacement, therefore all partial derivatives with respect to $u_{2}$ are zero and there is no propagation of displacement $u_{2}$ [2].

The state of deformation in the granular medium is described by the displacement vector $\underline{U}\left(u_{1}, 0, u_{3}\right)$ of the centre of gravity of a grain and the rotation vector $\underline{\xi}(\xi, \eta, \zeta)$ of the grain about its centre of gravity. There exist a stress tensor and a stress couple and are non-symmetric, that is,

$$
\tau_{i j} \neq \tau_{j i}, \quad M_{i j} \neq M_{j i}, \quad i=1,2,3 .
$$

The stress tensor $\tau_{i j}$ can be expressed as the sum of symmetric and anti-symmetric tensors

$$
\tau_{i j}=\sigma_{i j}+\sigma_{i j}^{\prime}
$$

where

$$
\sigma_{i j}=\frac{1}{2}\left(\tau_{i j}+\tau_{j i}\right), \quad \sigma_{i j}^{\prime}=\frac{1}{2}\left(\tau_{i j}-\tau_{j i}\right) .
$$

The symmetric tensor $\sigma_{i j}=\sigma_{j i}$ is related to the symmetric strain tensor

$$
e_{i j}=e_{j i}=\frac{1}{2}\left(\frac{\partial u_{i}}{\partial x_{j}}+\frac{\partial u_{j}}{\partial x_{i}}\right)
$$

by the Hook's law. 
The anti-symmetric stress $\sigma_{i j}^{\prime}$ are given by

$$
\begin{gathered}
\sigma_{23}^{\prime}=-F \frac{\partial \xi}{\partial t}, \quad \sigma_{31}^{\prime}=-F \frac{\partial \eta}{\partial t}, \quad \sigma_{12}^{\prime}=-F \frac{\partial \zeta}{\partial t}, \\
\sigma_{11}^{\prime}=\sigma_{22}^{\prime}=\sigma_{33}^{\prime}=0,
\end{gathered}
$$

where $F$ is the coefficient of fraction.

The stress couple $M_{i j}$ is given by

$$
M_{i j}=M v_{i j}
$$

where $M$ is the third elastic constant,

$$
\begin{gathered}
\nu_{11}=\frac{\partial \xi}{\partial x_{1}}, \quad v_{22}=0, \quad \nu_{33}=\frac{\partial \zeta}{\partial x_{3}}, \quad \nu_{23}=0, \\
v_{31}=\frac{\partial \xi}{\partial x_{3}}, \quad v_{12}=\frac{\partial}{\partial x_{1}}\left(\eta+\omega_{2}\right), \quad \nu_{32}=\frac{\partial}{\partial x_{3}}\left(\eta+\omega_{2}\right), \\
v_{13}=\frac{\partial \zeta}{\partial x_{1}}, \quad \nu_{21}=0,
\end{gathered}
$$

where $\omega_{2}=\partial u_{1} / \partial x_{3}-\partial u_{3} / \partial x_{1}$.

If $g$ is the acceleration due to gravity, then the components of body forces are $X=0$, $Z=g$. Assuming that the initial stress field due to gravity is hydrostatic, the states of initial stress $\tau_{i j}$ are [10]

$$
\begin{aligned}
\tau_{i j} & =\tau, \quad i=j, \\
\tau_{i j} & =0, \quad i \neq j,
\end{aligned}
$$

where $\tau$ is a function of depth $O x_{3}$ only.

The equilibrium conditions of the initial stress field are [10]

$$
\frac{\partial \tau}{\partial x_{1}}=\frac{\partial \tau}{\partial x_{2}}=0, \quad \frac{\partial \tau}{\partial x_{3}}+\rho g=0
$$

where $\rho$ is the density of the material medium. 
3148 Stoneley waves in granular medium

The six equations of motion are $[2,5]$

$$
\begin{gathered}
\frac{\partial \tau_{11}}{\partial x_{1}}+\frac{\partial \tau_{13}}{\partial x_{3}}+\rho g \frac{\partial u_{3}}{\partial x_{1}}=\rho \frac{\partial^{2} u_{1}}{\partial t^{2}} \\
\frac{\partial \tau_{12}}{\partial x_{1}}+\frac{\partial \tau_{32}}{\partial x_{3}}=0 \\
\frac{\partial \tau_{13}}{\partial x_{1}}+\frac{\partial \tau_{33}}{\partial x_{3}}-\rho g \frac{\partial u_{1}}{\partial x_{1}}=\rho \frac{\partial^{2} u_{3}}{\partial t^{2}}, \\
\tau_{23}-\tau_{32}+\frac{\partial M_{11}}{\partial x_{1}}+\frac{\partial M_{31}}{\partial x_{3}}=0 \\
\tau_{31}-\tau_{13}+\frac{\partial M_{12}}{\partial x_{1}}+\frac{\partial M_{32}}{\partial x_{3}}=0 \\
\tau_{12}-\tau_{21}+\frac{\partial M_{13}}{\partial x_{1}}+\frac{\partial M_{33}}{\partial x_{3}}=0
\end{gathered}
$$

These equations, when the stresses are substituted, take the forms

$$
\begin{gathered}
\frac{\partial}{\partial x_{1}}\left[C_{11} \frac{\partial u_{1}}{\partial x_{1}}+C_{13} \frac{\partial u_{3}}{\partial x_{3}}\right]+\frac{\partial}{\partial x_{3}}\left[C_{55}\left(\frac{\partial u_{3}}{\partial x_{1}}+\frac{\partial u_{1}}{\partial x_{3}}\right)-F \frac{\partial \eta}{\partial t}\right]+\rho g \frac{\partial u_{3}}{\partial x_{1}}=\rho \frac{\partial^{2} u_{1}}{\partial t^{2}} \\
\frac{\partial}{\partial x_{1}}\left(-F \frac{\partial \zeta}{\partial t}\right)+\frac{\partial}{\partial x_{3}}\left(F \frac{\partial \xi}{\partial t}\right)=0 \\
\frac{\partial}{\partial x_{1}}\left[C_{55}\left(\frac{\partial u_{3}}{\partial x_{1}}+\frac{\partial u_{1}}{\partial x_{3}}\right)+F \frac{\partial \eta}{\partial t}\right]+\frac{\partial}{\partial x_{3}}\left[C_{13} \frac{\partial u_{1}}{\partial x_{1}}+C_{33} \frac{\partial u_{3}}{\partial x_{3}}\right]-\rho g \frac{\partial u_{1}}{\partial x_{1}}=\rho \frac{\partial^{2} u_{3}}{\partial t^{2}} \\
-F \frac{\partial \xi}{\partial t}+\nabla^{2}(M \xi)=0 \\
-F \frac{\partial \eta}{\partial t}+\nabla^{2}\left[M\left(\eta+\frac{\partial u_{1}}{\partial x_{3}}-\frac{\partial u_{3}}{\partial x_{1}}\right)\right]=0 \\
-F \frac{\partial \zeta}{\partial t}+\nabla^{2}(M \zeta)=0
\end{gathered}
$$

where $C_{i j}$ are elastic constants.

\section{Solution of the problem}

We assume that the non-homogeneities are of the form

$$
C_{i j}=a_{i j} e^{m x_{3}}, \quad \rho=\rho_{0} e^{m x_{3}}, \quad F=F_{0} e^{m x_{3}}, \quad M=M_{0} e^{m x_{3}},
$$

where $a_{i j}, \rho_{0}, F_{0}, M_{0}$, and $m$ are constants. 
Substituting from (3.1) into (2.11), we get

$$
\begin{gathered}
a_{11} \frac{\partial^{2} u_{1}}{\partial x_{1}^{2}}+a_{13} \frac{\partial^{2} u_{3}}{\partial x_{1} \partial x_{3}}+a_{55}\left(\frac{\partial^{2} u_{3}}{\partial x_{1} \partial x_{3}}+\frac{\partial^{2} u_{1}}{\partial x_{3}^{2}}\right) \\
+m\left[a_{55}\left(\frac{\partial u_{3}}{\partial x_{1}}+\frac{\partial u_{1}}{\partial x_{3}}\right)-F_{0} \frac{\partial \eta}{\partial t}\right]-F_{0} \frac{\partial}{\partial t}\left(\frac{\partial \eta}{\partial x_{3}}\right)+\rho_{0} g \frac{\partial u_{3}}{\partial x_{1}}=\rho_{0} \frac{\partial^{2} u_{1}}{\partial t^{2}} \\
\frac{\partial}{\partial t}\left(m \xi+\frac{\partial \xi}{\partial x_{3}}-\frac{\partial \zeta}{\partial x_{1}}\right)=0 \\
a_{55}\left(\frac{\partial^{2} u_{3}}{\partial x_{1}^{2}}+\frac{\partial^{2} u_{1}}{\partial x_{1} \partial x_{3}}\right)+a_{13} \frac{\partial^{2} u_{1}}{\partial x_{1} \partial x_{3}}+a_{33} \frac{\partial^{2} u_{3}}{\partial x_{3}^{2}} \\
+m\left(a_{31} \frac{\partial u_{1}}{\partial x_{1}}+a_{33} \frac{\partial u_{3}}{\partial x_{3}}\right)+F_{0} \frac{\partial}{\partial t}\left(\frac{\partial n}{\partial x_{1}}\right)-\rho_{0} g \frac{\partial u_{1}}{\partial x_{1}}=\rho_{0} \frac{\partial^{2} u_{3}}{\partial t^{2}} \\
-F_{0} \frac{\partial \xi}{\partial t}+M_{0} \nabla^{2} \xi+m M_{0} \frac{\partial \xi}{\partial x_{3}}=0 \\
-F_{0} \frac{\partial \zeta}{\partial t}+M_{0} \nabla^{2} \zeta+m M_{0} \frac{\partial \zeta}{\partial x_{3}}=0 . \\
-F_{0} \frac{\partial \eta}{\partial t}+M_{0} \nabla^{2}\left(\eta+\frac{\partial u_{1}}{\partial x_{3}}-\frac{\partial u_{3}}{\partial x_{1}}\right)+m M_{0} \frac{\partial}{\partial x_{3}}\left(\eta+\frac{\partial u_{1}}{\partial x_{3}}-\frac{\partial u_{3}}{\partial x_{1}}\right)=0 \\
+
\end{gathered}
$$

We assume that the displacements $u_{1}$ and $u_{3}$ are derivable from the displacement potentials $\phi\left(x_{1}, x_{3}, t\right), \psi\left(x_{1}, x_{3}, t\right)$ by the relations

$$
u_{1}=\frac{\partial \phi}{\partial x_{1}}-\frac{\partial \psi}{\partial x_{3}}, \quad u_{3}=\frac{\partial \phi}{\partial x_{3}}+\frac{\partial \psi}{\partial x_{1}}
$$

Substituting from (3.3) into (3.2), we get the following wave equations satisfied by $\phi$, $\psi, \xi, \eta$, and $\zeta$ :

$$
\begin{gathered}
a_{11} \frac{\partial^{2} \phi}{\partial x_{1}^{2}}+\left(a_{13}+2 a_{55}\right) \frac{\partial^{2} \phi}{\partial x_{3}^{2}}+2 m a_{55} \frac{\partial \phi}{\partial x_{3}}+\left(m a_{55}+\rho_{0} g\right) \frac{\partial \psi}{\partial x_{1}}=\rho_{0} \frac{\partial^{2} \phi}{\partial t^{2}}, \\
\frac{\partial}{\partial t}\left(m \xi+\frac{\partial \xi}{\partial x_{3}}-\frac{\partial \zeta}{\partial x_{1}}\right)=0, \\
a_{55} \frac{\partial^{2} \psi}{\partial x_{1}^{2}}+\left(a_{33}-a_{31}-a_{55}\right) \frac{\partial^{2} \psi}{\partial x_{3}^{2}}+m a_{33} \frac{\partial \psi}{\partial x_{3}}+\left(m a_{31}-\rho_{0} g\right) \frac{\partial \phi}{\partial x_{1}}+F_{0} \frac{\partial \eta}{\partial t}=\rho_{0} \frac{\partial^{2} \psi}{\partial t^{2}},
\end{gathered}
$$


3150 Stoneley waves in granular medium

$$
\begin{gathered}
\nabla^{2} \xi+m \frac{\partial \xi}{\partial x_{3}}-S_{0} \frac{\partial \xi}{\partial t}=0 \\
\nabla^{2} \eta+m \frac{\partial \eta}{\partial x_{3}}-S_{0} \frac{\partial \eta}{\partial t}-\nabla^{4} \psi-m \frac{\partial}{\partial x_{3}}\left(\nabla^{2} \psi\right)=0 \\
\nabla^{2} \zeta+m \frac{\partial \zeta}{\partial x_{3}}-S_{0} \frac{\partial \zeta}{\partial t}=0
\end{gathered}
$$

where $S_{0}=F_{0} / M_{0}$.

Eliminating $\eta$ from (3.6) and (3.8), we get

$$
\begin{aligned}
F_{0} \nabla^{4}\left(\frac{\partial \psi}{\partial t}\right)+m F_{0} \frac{\partial^{2}}{\partial x_{3} \partial t}(\nabla \psi) & \\
+\left(\nabla^{2}+m \frac{\partial}{\partial x_{3}}-S_{0} \frac{\partial}{\partial t}\right) & {\left[a_{55} \frac{\partial^{2} \psi}{\partial x_{1}^{2}}+\left(a_{33}-a_{31}-a_{55}\right) \frac{\partial^{2} \psi}{\partial x_{3}^{2}}\right.} \\
& \left.+m a_{33} \frac{\partial \psi}{\partial x_{3}}+\left(m a_{31}-\rho_{0} g\right) \frac{\partial \phi}{\partial x_{1}}-\rho_{0} \frac{\partial^{2} \psi}{\partial t^{2}}\right]=0 .
\end{aligned}
$$

Assuming that

$$
\begin{aligned}
(\phi, \psi) & =\left\{\phi_{1}\left(x_{3}\right), \psi_{1}\left(x_{3}\right)\right\} \exp \left\{i\left(L x_{1}-b t\right)\right\}, \\
(\xi, \eta, \zeta) & =\left\{\xi_{1}\left(x_{3}\right), \eta_{1}\left(x_{3}\right), \zeta\left(x_{3}\right)\right\} \exp \left\{i\left(L x_{1}-b t\right)\right\} .
\end{aligned}
$$

Substituting from (3.11) into (3.4) and (3.10), we get

$$
\begin{aligned}
& \quad\left(\left(a_{13}+2 a_{55}\right) D^{2}+2 m a_{55} D-a_{11} L^{2}+\rho_{0} b^{2}\right) \phi_{1}+i L\left(m a_{55}+\rho_{0} g\right) \psi_{1}=0, \\
& \left\{\left[\left(a_{33}-a_{31}-a_{55}\right)-i b F_{0}\right] D^{4}+m\left[\left(2 a_{33}-a_{31}-a_{55}\right)-i b F_{0}\right] D^{3}\right. \\
& \quad-\left[\left(L^{2} a_{55}-\rho_{0} b^{2}+m^{2} a_{33}-2 i b L F_{0}\right)+\left(L^{2}-i b s_{0}\right)\left(a_{33}-a_{31}-a_{55}\right)\right] D^{2} \\
& \quad-m\left(\left(L^{2} a_{55}-\rho_{0} b^{2}\right)+a_{33}\left(L^{2}-i b S_{0}\right)-i b F_{0} L^{2}\right) D \\
& \left.\quad+\left(\left(L^{2}-i b S_{0}\right)\left(L^{2} a_{55}-\rho_{0} b^{2}\right)-i b F_{0} L^{4}\right)\right\} \psi_{1} \\
& \quad-i L\left(\rho_{0} g-m a_{31}\right)\left(D^{2}+m D-\left(L^{2}-i b S_{0}\right)\right) \phi_{1}=0,
\end{aligned}
$$

where $D \equiv d / d x_{3}$.

Equations (3.13) and (3.14) must have exponential solutions in order that $\phi_{1}, \psi_{1}$ will describe surface waves; they must become vanishingly small as $x_{3} \rightarrow \infty$. Hence, for the medium $M_{1}$,

$$
\begin{gathered}
\phi_{1}=A_{j} e^{-\lambda_{j} x_{3}}, \\
\psi_{1}=B_{j} e^{-\lambda_{j} x_{3}}, \quad(j=3,4,5),
\end{gathered}
$$

where the constants $A_{j}$ are related with the constants $B_{j}$, respectively, by means of (3.13). 
Equating the coefficients of the exponentials $e^{-\lambda_{j} x_{3}}(j=3,4,5)$ to zero and using $(3.13)$ and (3.14), we have

$$
A_{j}=n_{j} B_{j}
$$

where

$$
n_{j}=\frac{-i L\left(\rho_{0} g+m a_{55}\right)}{\left(a_{13}+2 a_{55}\right) \lambda_{j}^{2}-2 m a_{55} \lambda_{j}+\rho_{0} b^{2}-a_{11} L^{2}}, \quad(j=3,4,5),
$$

$\lambda_{3}, \lambda_{4}, \lambda_{5}$ are the roots which have a positive real part of the equation

$$
\begin{aligned}
k_{0} \lambda^{6}+ & k_{1} \lambda^{5}+k_{2} \lambda^{4}+k_{3} \lambda^{3}+k_{4} \lambda^{2}+k_{5} \lambda+k 6=0, \\
k_{0}= & \left(a_{13}+2 a_{55}\right)\left[\left(a_{33}-a_{31}-a_{55}\right)-i b F_{0}\right], \\
k_{1}= & -m\left\{\left(a_{13}+4 a_{55}\right)\left[\left(a_{33}-a_{31}-a_{55}\right)-i b F_{0}\right]+a_{33}\left(a_{13}+2 a_{55}\right)\right\}, \\
k_{2}= & {\left[\left(a_{33}-a_{31}-a_{55}\right)-i b F_{0}\right]\left(\rho_{0} b^{2}-a_{11} L^{2}+2 m^{2} a_{55}\right)+2 m^{2} a_{33} a_{55} } \\
& -\left(a_{13}+2 a_{55}\right)\left(L^{2} a_{55}-\rho_{0} b^{2}+m^{2} a_{33}-2 i b L^{2} F_{0}+\left(L^{2}-i b S_{0}\right)\left(a_{33}-a_{31}-a_{55}\right)\right), \\
k_{3}= & -m\left\{\left(\rho_{0} b^{2}-a_{11} L^{2}\right)\left[\left(2 a_{33}-a_{31}-a_{55}\right)-i b F_{0}\right]\right. \\
& \quad-2 a_{55}\left(L^{2} a_{55}-\rho_{0} b^{2}+m^{2} a_{33}-2 i b L^{2} F_{0}+\left(L^{2}-i b S_{0}\right)\left(a_{33}-a_{31}-a_{55}\right)\right) \\
& \left.\quad-\left(a_{13}+2 a_{55}\right)\left(L^{2} a_{55}-\rho_{0} b^{2}-i b L^{2} F_{0}+a_{33}\left(L^{2}-i b S_{0}\right)\right)\right\}, \\
k_{4}= & \left(a_{11} L^{2}-\rho_{0} b^{2}\right)\left(L^{2} a_{55}-\rho_{0} b^{2}+m^{2} a_{33}-2 i b L^{2} F_{0}+\left(L^{2}-i b S_{0}\right)\left(a_{33}-a_{31}-a_{55}\right)\right) \\
& -2 m^{2} a_{55}\left[\left(L^{2} a_{55}-\rho_{0} b^{2}\right)+a_{33}\left(L^{2}-i b S_{0}\right)-i b F_{0} L^{2}\right] \\
& -\left(a_{13}+2 a_{55}\right)\left[\left(L^{2}-i b S_{0}\right)\left(\rho_{0} b^{2}-L^{2} a_{55}\right)+i b F_{0} L^{4}\right] \\
& +L^{2}\left(m a_{55}+\rho_{0} g\right)\left(m a_{31}-\rho_{0} g\right), \\
k_{5}= & m\left\{L^{2} a_{55}-\rho_{0} b^{2}+a_{33}\left(L^{2}-i b S_{0}\right)-i b F_{0} L^{2}\right. \\
& \left.+2 a_{55}\left(\left(L^{2}-i b S_{0}\right)\left(\rho_{0} b^{2}-L^{2} a_{55}\right)+i b F_{0} L^{4}\right)-L^{2}\left(m a_{55}+\rho_{0} g\right)\left(m a_{31}-\rho_{0} g\right)\right\}, \\
k_{6}= & \left(a_{11} L^{2}-\rho_{0} b^{2}\right)\left(\left(L^{2}-i b S_{0}\right)\left(\rho_{0} b^{2}-a_{55} L^{2}\right)+i b F_{0} L^{4}\right) \\
& -L^{2}\left(m a_{55}+\rho_{0} g\right)\left(m a_{31}-\rho_{0} g\right)\left(L^{2}-i b S_{0}\right) .
\end{aligned}
$$

Using (3.8), (3.11), (3.12), and (3.16), one gets

$$
\eta_{1}=\Omega_{j}\left(B_{j} e^{-\lambda_{j} x_{3}}\right)
$$


3152 Stoneley waves in granular medium

where

$$
\Omega_{j}=\frac{\lambda_{j}^{4}-m \lambda_{j}^{3}-2 L^{2} \lambda_{j}^{4}+m L^{2} \lambda_{i}+L^{4}}{\lambda_{j}^{2}-m \lambda_{i}+i b S_{0}-L^{2}} .
$$

Also, substituting from (3.12) into (3.5), (3.7), and (3.9), we get

$$
\begin{aligned}
& (D+m) \xi_{1}-i L \zeta_{1}=0 \\
& \left(D^{2}+m D+h^{2}\right) \xi_{1}=0 \\
& \left(D^{2}+m D+h^{2}\right) \zeta_{1}=0
\end{aligned}
$$

where $h^{2}=i b S_{0}-L^{2}$.

The solutions of (3.23) and (3.24) are

$$
\xi_{1}=A_{2} e^{-i h_{2} x_{3}}, \quad \zeta_{1}=B_{2} e^{-i h_{2} x_{3}},
$$

where $h_{2}=\left(-m+\sqrt{m^{2}-4 h^{2}}\right) / 2$.

From (3.25) and (3.22), one can obtain

$$
A_{2}=\frac{-L}{h_{2}+i m} B_{2}
$$

We use the symbols with a bar for the upper medium (except $x_{3}, L, b, g$ ) and the functions $\bar{\xi}_{1}, \bar{\zeta}_{1}, \bar{\eta}_{1}, \bar{\phi}_{1}$, and $\bar{\psi}_{1}$ must vanish as $x \rightarrow-\infty$.

For the upper medium $M_{2}$, we have

$$
\begin{gathered}
\bar{\xi}_{1}=\bar{A}_{2} e^{i \bar{h}_{2} x_{3}}, \quad \bar{\zeta}_{1}=\bar{B}_{2} e^{i \bar{h}_{2} x_{3}}, \\
\bar{\eta}_{1}=\bar{\Omega}_{j} \bar{B}_{j} e^{\bar{\lambda}_{j} x_{3}}, \quad \bar{\phi}_{1}=\bar{A}_{j} e^{\bar{\lambda}_{j} x_{3}}, \quad \bar{\psi}_{1}=\bar{B}_{j} e^{\bar{\lambda}_{j} x_{3}} \quad(j=3,4,5) .
\end{gathered}
$$

\section{Boundary conditions and frequency equation}

The boundary conditions on the interface $x_{3}=0$ are
(i) $u_{1}=\bar{u}_{1}$,
(ii) $u_{3}=\bar{u}_{3}$,
(iii) $\xi=\bar{\xi}$,
(iv) $\eta=\bar{\eta}$,
(v) $\zeta=\bar{\zeta}$,
(vi) $M_{33}=\bar{M}_{33}$,
(vii) $M_{31}=\bar{M}_{31}$,
(viii) $M_{32}=\bar{M}_{32}$,
(ix) $\tau_{33}=\bar{\tau}_{33}$,
(x) $\tau_{31}=\bar{\tau}_{31}$,
(xi) $\tau_{32}=\bar{\tau}_{32}$, 
where

$$
\begin{gathered}
M_{33}=M \frac{\partial \zeta}{\partial x_{3}}, \quad M_{32}=M \frac{\partial}{\partial x_{3}}\left(\eta-\nabla^{2} \psi\right), \quad M_{31}=M \frac{\partial \xi}{\partial x_{3}} \\
\tau_{33}=C_{13} \frac{\partial^{2} \phi}{\partial x_{1}^{2}}+C_{33} \frac{\partial^{2} \phi}{\partial x_{3}^{2}}+\left(C_{33}-C_{13}\right) \frac{\partial^{2} \psi}{\partial x_{1} \partial x_{3}}, \\
\tau_{32}=-F \frac{\partial \xi}{\partial t}, \quad \tau_{31}=C_{55}\left(\frac{\partial^{2} \psi}{\partial x_{1}^{2}}-\frac{\partial^{2} \psi}{\partial x_{3}^{2}}+2 \frac{\partial^{2} \phi}{\partial x_{1} \partial x_{3}}\right)-F \frac{\partial \eta}{\partial t}
\end{gathered}
$$

From the boundary conditions (iii), (v), (vi), and (vii), we get

$$
A_{2}=\bar{A}_{2}, \quad B_{2}=\bar{B}_{2}, \quad h_{2} M_{0}=-\bar{h}_{2} \bar{M}_{0}
$$

whence $A_{2}=\bar{A}_{2}=B_{2}=\bar{B}_{2}=0, \xi=\zeta=\bar{\xi}=\bar{\zeta}=0$.

The other significant boundary conditions are responsible for the following relations:

(i) $\left(i L n_{j}+\lambda_{j}\right) B_{j}=\left(i L \bar{n}_{j}-\bar{\lambda}_{j}\right) \bar{B}_{j}$,

(ii) $\left(i L-n_{j} \lambda_{j}\right) B_{j}=\left(i L+\bar{n}_{j} \bar{\lambda}_{j}\right) \bar{B}_{j}$,

(iv) $\Omega_{j} B_{j}=\bar{\Omega}_{j} \bar{B}_{j}$,

(viii) $M_{0}\left[\left(L^{2}+\Omega_{j}\right) \lambda_{j}-\lambda_{j}^{3}\right] B_{j}=\bar{M}_{0}\left[-\left(L^{2}+\bar{\Omega}_{j}\right) \bar{\lambda}_{j}+\bar{\lambda}_{j}^{3}\right] \bar{B}_{j}$,

(ix) $\left[\left(a_{33} \lambda_{j}^{2}-a_{13} L^{2}\right) n_{j}-i L\left(a_{33}-a_{13}\right) \lambda_{i}\right] B_{j}=\left[\left(\bar{a}_{33} \bar{\lambda}_{j}^{2}-\bar{a}_{13} L^{2}\right) \bar{n}_{j}+i L\left(\bar{a}_{33}-\bar{a}_{13}\right) \bar{\lambda}_{i}\right] \bar{B}_{j}$,

(x) $\left[a_{55}\left(L^{2}+\lambda_{j}^{2}+2 i L n_{j} \lambda_{j}\right)-i b F_{0} \Omega_{j}\right] B_{j}=\left[\bar{a}_{55}\left(L^{2}+\bar{\lambda}_{j}^{2}-2 i L \bar{n}_{j} \bar{\lambda}_{j}\right)-i b \bar{F}_{0} \bar{\Omega}_{j}\right] \bar{B}_{j}$.

Eliminating the constants $B_{j}, \bar{B}_{j}(j=3,4,5)$, we obtain the wave velocity equation in the form of a sixth-order determinantal equation,

$\left|\begin{array}{cccccc}i L n_{3}+\lambda_{3} & i L n_{4}+\lambda_{4} & i L n_{5}+\lambda_{5} & i L \bar{n}_{3}-\bar{\lambda}_{3} & i L \bar{n}_{4}-\bar{\lambda}_{4} & i L \bar{n}_{5}-\bar{\lambda}_{5} \\ i L-n_{3} \lambda_{3} & i L-n_{4} \lambda_{4} & i L-n_{5} \lambda_{5} & i L+\bar{n}_{3} \bar{\lambda}_{3} & i L+\bar{n}_{4} \bar{\lambda}_{4} & i L+\bar{n}_{5} \bar{\lambda}_{5} \\ \Omega_{3} & \Omega_{4} & \Omega_{5} & \bar{\Omega}_{3} & \bar{\Omega}_{4} & \bar{\Omega}_{5} \\ Q_{13} & Q_{14} & Q_{15} & \bar{Q}_{13} & \bar{Q}_{14} & \bar{Q}_{15} \\ Q_{23} & Q_{24} & Q_{25} & \bar{Q}_{23} & \bar{Q}_{24} & \bar{Q}_{25} \\ Q_{33} & Q_{34} & Q_{35} & \bar{Q}_{33} & \bar{Q}_{34} & \bar{Q}_{35}\end{array}\right|=0$,

where

$$
\begin{aligned}
& Q_{1 j}=M_{0}\left(L^{2}+\Omega_{j}-\lambda_{j}^{2}\right) \lambda_{j}, \\
& Q_{2 j}=n_{j}\left(a_{33} \lambda_{j}^{2}-a_{13} L^{2}\right)-i L\left(a_{33}-a_{13}\right) \lambda_{j}, \\
& Q_{3 j}=a_{55}\left(L^{2}+\lambda_{j}^{2}+2 i L n_{j} \lambda_{j}\right)-i b F_{0} \Omega_{j}, \\
& \bar{Q}_{1 j}=-\bar{M}_{0}\left(L^{2}+\bar{\Omega}_{j}-\bar{\lambda}_{j}^{2}\right) \bar{\lambda}_{j}, \\
& \bar{Q}_{2 j}=i L\left(a_{33}-a_{13}\right) \bar{\lambda}_{j}-\bar{n}_{j}\left(a_{13} L^{2}-a_{33} \bar{\lambda}_{j}^{2}\right), \\
& \bar{Q}_{3 j}=\bar{a}_{55}\left(L^{2}-2 i L \bar{n}_{j} \bar{\lambda}_{j}+\bar{\lambda}_{j}^{2}\right)-i b \bar{F}_{0} \bar{\Omega}_{j}, \quad j=3,4,5 .
\end{aligned}
$$

Equation (4.3) is the frequency equation of Stoneley waves in a non-homogeneous orthotropic granular medium under the influence of gravity, this equation depends on the 
3154 Stoneley waves in granular medium

particular values of $\lambda_{j}$ and $\bar{\lambda}_{j}$ creating a dispersion of the general wave form. Moreover, the wave velocity $C(=b / L)$ depends on the gravity field, the non-homogeneous of the material medium and the granular rotations.

From (3.18), (3.19), and (4.3), we can assert that when $L$ is Large, so that the length of the wave is small, the effect of gravity is sufficiently small, that is, the wave length of the wave is large, the effect of gravity is no longer negligible and plays an important role on the determination of the wave velocity $C$.

If we neglect the gravity field, we obtain the wave velocity equation for Stoneley waves in a non-homogeneous orthotropic granular medium which is the same equation as (4.3) with

$$
n_{j}=\frac{-i m L a_{55}}{\left(a_{13}+2 a_{55}\right) \lambda_{i}^{2}-2 m a_{55} \lambda_{i}+\rho_{0} b^{2}-a_{11} L^{2}}, \quad(j=3,4,5),
$$

where $\lambda_{j}$ are the roots of the equation

$$
\begin{aligned}
k_{0} \lambda^{6}+ & k_{1} \lambda^{5}+k_{2} \lambda^{4}+k_{3} \lambda^{3}+k_{4}^{\prime} \lambda^{2}+k_{5}^{\prime} \lambda+k_{6}^{\prime}=0, \\
k_{4}^{\prime}= & \left(a_{11} L^{2}-\rho_{0} b^{2}\right)\left[L^{2} a_{55}-\rho_{0} b^{2}+m^{2} a_{33}-2 i b L^{2} F_{0}+\left(L^{2}-i b s_{0}\right)\left(a_{33}-a_{31}-a_{55}\right)\right] \\
& -2 m^{2} a_{55}\left[\left(L^{2} a_{55}-\rho_{0} b^{2}\right)+a_{33}\left(L^{2}-i b s_{0}\right)-i b F_{0} L^{2}\right] \\
& \quad-\left(a_{13}+2 a_{55}\right)\left[\left(L^{2}-i b s_{0}\right)\left(\rho_{0} b^{2}-L^{2} a_{55}\right)+i b F_{0} L^{4}\right]+m^{2} L^{2} a_{55} a_{31}, \\
k_{5}^{\prime}= & m\left\{L^{2} a_{55}-\rho_{0} b^{2}+a_{33}\left(L^{2}-i b s_{0}\right)-i b F_{0} L^{2}\right. \\
& \left.\quad+2 a_{55}\left[\left(L^{2}-i b s_{0}\right)\left(\rho_{0} b^{2}-L^{2} a_{55}\right)+i b F_{0} L^{4}\right]-m^{2} L^{2} a_{55} a_{31}\right\}, \\
k_{6}^{\prime}= & \left(a_{11} L^{2}-\rho_{0} b^{2}\right)\left[\left(L^{2}-i b s_{0}\right)\left(\rho_{0} b^{2}-a_{55} L^{2}\right)+i b F_{0} L^{4}\right]-m^{2} L^{2} a_{55} a_{31}\left(L^{2}-i b s_{0}\right) .
\end{aligned}
$$

When both media are elastic $\left(M_{0}=0, F_{0}=0\right)$, by using (3.4) and (3.6); (3.18) becomes

$$
n_{j}=\frac{-i L\left(m a_{55}+\rho_{0} g\right)}{\left(a_{13}+2 a_{55}\right) \lambda_{j}^{2}-2 m a_{55} \lambda_{j}-a_{11} L^{2}+\rho_{0} b^{2}}, \quad(j=3,4)
$$

$\lambda_{j}$ are the real roots of the equation

$$
\begin{aligned}
& \left(a_{13}+2 a_{55}\right)\left(a_{33}-a_{31}-a_{55}\right) \lambda^{4} \\
& \quad-m\left[2 a_{55}\left(a_{33}-a_{31}-a_{55}\right)+a_{33}\left(a_{13}+2 a_{55}\right)\right] \lambda^{3} \\
& \quad+\left[\left(\rho_{0} b^{2}-a_{11} L^{2}\right)\left(a_{33}-a_{31}-a_{55}\right)+\left(a_{13}+2 a_{55}\right)\left(\rho_{0} b^{2}-a_{55} L^{2}\right)+2 m^{2} a_{55} a_{33}\right] \lambda^{2} \\
& \quad+m L^{2}\left[a_{33}\left(a_{11}-\rho_{0} b^{2}\right)+\left(a_{13}+2 a_{55}\right)\left(a_{55}-\rho_{0} b^{2}\right)\right] \lambda \\
& \quad+L^{2}\left[\left(\rho_{0} b^{2}-a_{11}\right)\left(\rho_{0} b^{2}-a_{55}\right)+\left(m a_{31}-\rho_{0} g\right)\left(m a_{55}+\rho_{0} g\right)\right]=0,
\end{aligned}
$$


and the frequency equation (4.3) takes the form

$$
\left|\begin{array}{cccc}
i L n_{3}+\lambda_{3} & i L n_{4}+\lambda_{4} & i L \bar{n}_{3}-\bar{\lambda}_{3} & i L \bar{n}_{4}-\bar{\lambda}_{4} \\
i L-n_{3} \lambda_{3} & i L-n_{4} \lambda_{4} & i L+\bar{n}_{3} \bar{\lambda}_{3} & i L+\bar{n}_{4} \bar{\lambda}_{4} \\
Q_{23} & Q_{24} & \bar{Q}_{23} & \bar{Q}_{24} \\
Q_{33} & Q_{34} & \bar{Q}_{33} & \bar{Q}_{34}
\end{array}\right|=0 .
$$

Equation (4.9) determines the wave velocity equation for Stoneley wave in a nonhomogeneous orthotropic elastic medium under the influence of gravity and is in complete agreement with that obtained by Abd-Alla and Ahmed [2].

\section{References}

[1] A. M. Abd-Alla and S. M. Ahmed, Rayleigh waves in an orthotropic thermoelastic medium under gravity field and initial stress, J. Earth, Moon Planets 75 (1996), no. 3, 185-197.

[2] Stoneley and Rayleigh waves in a non-homogeneous orthotropic elastic medium under the influence of gravity, Appl. Math. Comput. 135 (2003), no. 1, 187-200.

[3] S. M. Ahmed, Influence of gravity on the propagation of waves in granular medium, Appl. Math. Comput. 101 (1999), no. 2-3, 269-280.

[4] - Rayleigh waves in a thermoelastic granular medium under initial stress, Int. J. Math. Math. Sci. 23 (2000), no. 9, 627-637.

[5] R. K. Bhattacharyya, Rayleigh waves in granular medium, Pure Appl. Geophys. 62 (1965), no. 3, $13-22$.

[6] S. C. Das, D. P. Acharya, and P. R. Sengupta, Surface waves in an inhomogeneous elastic medium under the influence of gravity, Rev. Roumaine Sci. Tech. Sér. Méc. Appl. 37 (1992), no. 5, $539-551$.

[7] A. M. El-Naggar, On the dynamical problem of a generalized thermoelastic granular infinite cylinder under initial stress, Astrophys. Space Sci. 190 (1992), no. 2, 177-190.

[8] A. M. El-Naggar, A. M. Abd-Alla, and S. M. Ahmed, Rayleigh waves in a magnetoelastic initially stressed conducting medium with the gravity field, Bull. Calcutta Math. Soc. 86 (1994), no. 3 , 243-248.

[9] M. A. Goda, The effect of inhomogeneity and anisotropy on Stoneley waves, Acta Mech. 93 (1992), no. 1-4, 89-98.

[10] A. E. H. Love, Some Problems of Geodynamics, Dover, New York, 1965.

[11] N. Oshima, Mem. Unifying Study of Basic Problems in Engineering Sciences by Means of Geometry Vol. I, III, (General Editor, K. Kondo, 1955).

[12] - Proc. 3rd Japan Nat. Congr. Appl. Mech., 77, 1954.

[13] G. Paria, Love waves in granular medium, Bull. Calcutta Math. Soc. 52 (1960), no. 4, 195-203.

S. M. Ahmed: Mathematics Department, Faculty of Education, Suez Canal University, El-Arish, Egypt

E-mail address: saidmahmed@hotmail.com 


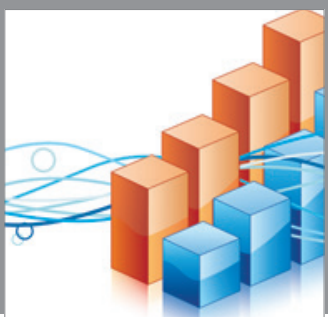

Advances in

Operations Research

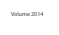

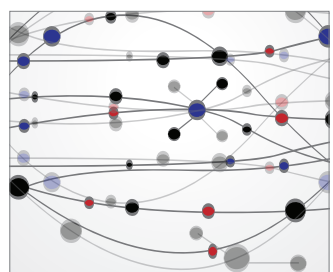

\section{The Scientific} World Journal
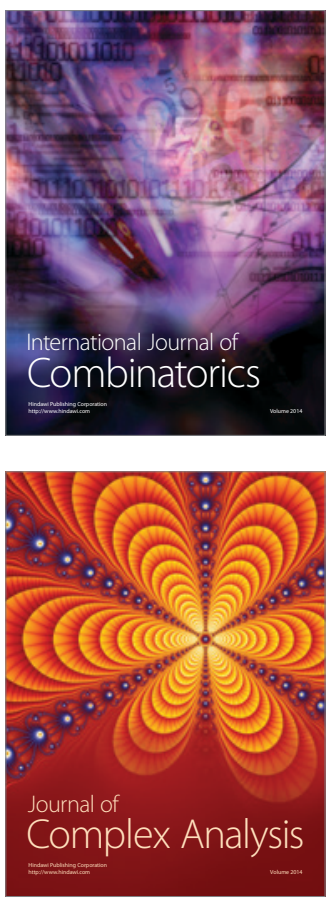

International Journal of

Mathematics and

Mathematical

Sciences
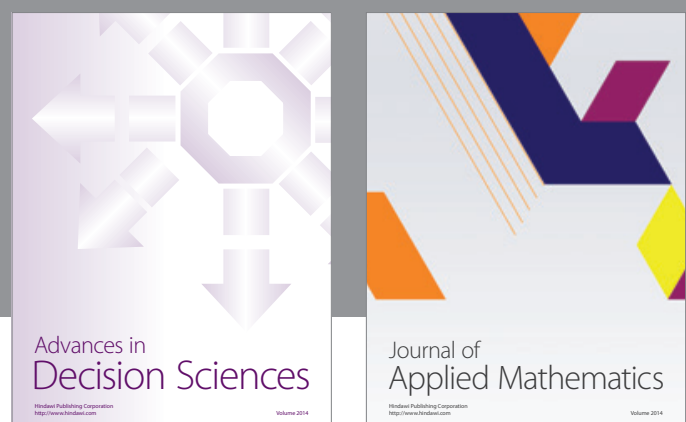

Journal of

Applied Mathematics
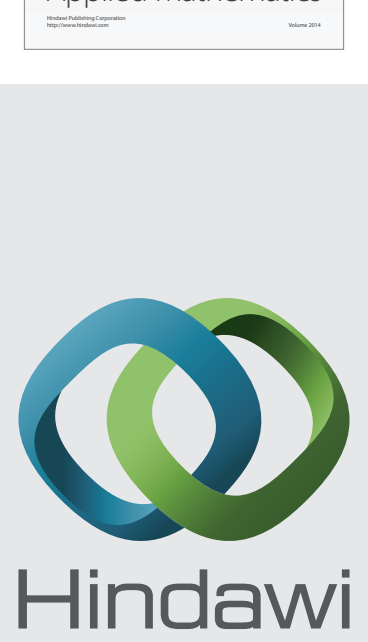

Submit your manuscripts at http://www.hindawi.com
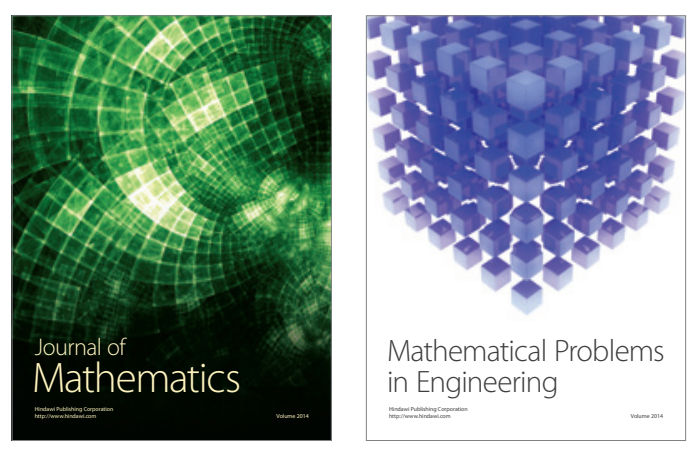

Mathematical Problems in Engineering
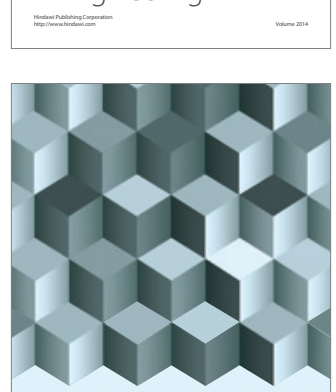

Journal of

Function Spaces
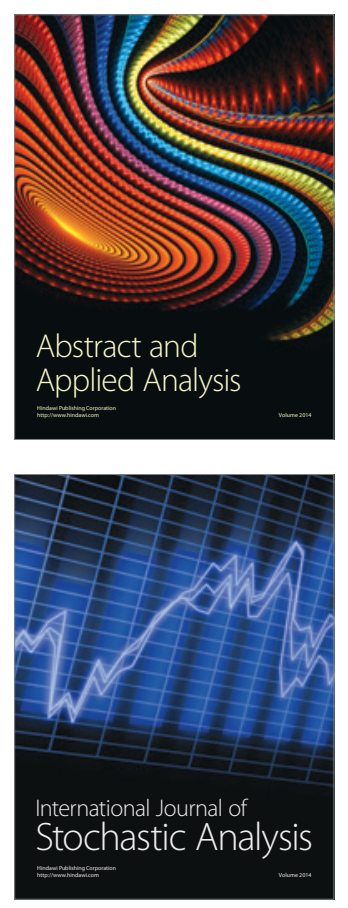

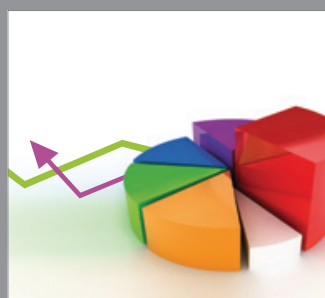

ournal of

Probability and Statistics

Promensencen
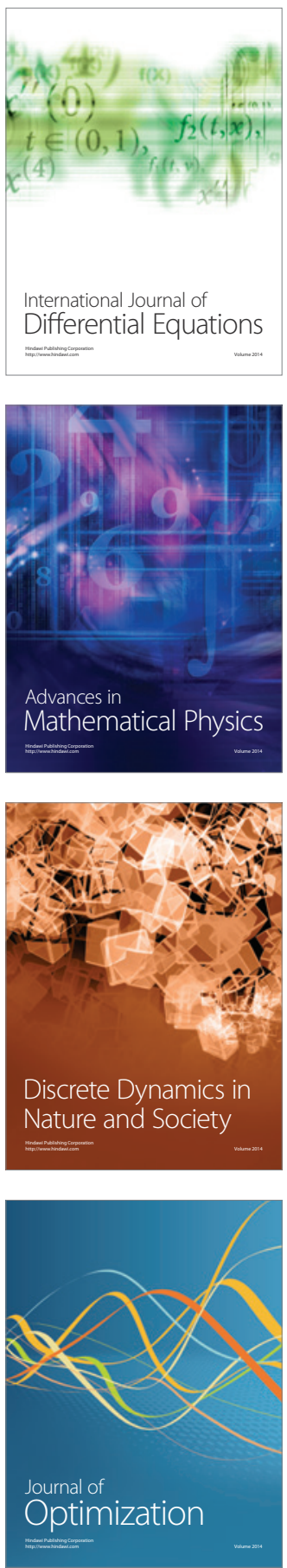